\title{
BMJ Open Study protocol for the ABERRANT study: antibiotic-induced disruption of the maternal and infant microbiome and adverse health outcomes - a prospective cohort study among children born at term
}

\author{
Maryse Volery, ${ }^{1,2}$ Valentin Scherz, ${ }^{3,4}$ William Jakob, ${ }^{5}$ Diane Bandeira, ${ }^{5}$ \\ Vanessa Deggim-Messmer, ${ }^{5}$ Anna Lauber-Biason, ${ }^{1,2}$ Johannes Wildhaber, ${ }^{1,2}$ \\ Laurent Falquet, ${ }^{6,7}$ Nigel Curtis, ${ }^{8,9,10}$ Petra Zimmermann (iD) ${ }^{1,2,8,9}$
}

To cite: Volery M, Scherz V, Jakob W, et al. Study protocol for the ABERRANT study: antibiotic-induced disruption of the maternal and infant microbiome and adverse health outcomes - a prospective cohort study among children born at term. BMJ Open 2020;10:e036275. doi:10.1136/ bmjopen-2019-036275

- Prepublication history for this paper is available online. To view these files, please visit the journal online (http://dx.doi org/10.1136/bmjopen-2019036275).

Received 08 December 2019 Revised 12 May 2020 Accepted 21 May 2020

A) Check for updates

(C) Author(s) (or their employer(s)) 2020. Re-use permitted under CC BY-NC. No commercial re-use. See rights and permissions. Published by BMJ.

For numbered affiliations see end of article.

Correspondence to Dr Petra Zimmermann; petra.zimmermann@unifr.ch

\section{ABSTRACT \\ Introduction There is compositional overlap between the maternal intestinal microbiome, the breast milk microbiome and the infant oral and intestinal microbiome. Antibiotics cause profound changes in the microbiome. However, the effect of intrapartum and early-life antibiotics on the maternal intestinal and breast milk microbiome, and the infant oral and intestinal microbiome, and whether effects are only short term or persist long term remain uncertain.}

Methods and analyses In this prospective cohort study, we will use metagenomic sequencing to determine: (1) the effect of intrapartum antibiotics on the composition of the breast milk, and the infant oral and intestinal microbiome, including the development and persistence of antibiotic resistance; (2) the effect of antibiotic exposure in the first year of life on the composition of the infant oral and intestinal microbiome, including the development and persistence of antibiotic resistance; (3) the effect of disruption of the infant oral and intestinal microbiome on health outcomes and (4) the compositional overlap between the maternal intestinal microbiome, the breast milk microbiome and the infant oral and intestinal microbiome.

Ethics and dissemination The ABERRANT study has been approved by the commission cantonale d'éthique de la recherche sur l'être humain (CER-VD) du Canton de Vaud (\#2019-01567). Outcomes will be disseminated through publication and will be presented at scientific conferences.

Trial registration number NCT04091282.

\section{INTRODUCTION}

Antibiotics are among the most commonly used drugs, especially in infants and children. Even before birth, more than $40 \%$ of infants are exposed to antibiotics. ${ }^{2}$ Additionally, more than two-thirds of children receive antibiotics before reaching the age of 2 years. ${ }^{34}$
Strengths and limitations of this study

- The use of metagenomic sequencing to comprehensively determine the effect of intrapartum and early-life antibiotics on the composition of the maternal breast milk and the infant oral and intestinal microbiome.

- The possibility to identify bacterial species, together with resistance genes and other important components of the microbiome such as archaea, eukaryotes (fungi) and viruses.

- The investigation of the association between the early-life intestinal microbiome and clinical health outcomes.

- The potential for contamination of low microbial biomass such as breast milk or meconium samples from the environment or extraction and sequencing kits.

The human intestine is the habitat for a large community of microbes, the intestinal microbiome. Colonisation of the intestinal tract increases rapidly after birth and the microbiome of the maternal intestine and breast milk are likely important source for the infant intestinal microbiome. ${ }^{5}$ Conversely, as retrograde flow of breast milk into mammary ducts has been documented ${ }^{6}$ the infant oral microbiome might be responsible for colonising the mammary ducts and therefore could contribute to the breast milk microbiome. Consequently, there is compositional overlap between the maternal intestinal microbiome, the breast milk microbiome and the infant oral and intestinal microbiome. ${ }^{78}$

Growing evidence shows that the composition of the intestinal microbiome in infants 
plays an important role in the development and regulation of the immune system, especially in the early-life 'critical window' during which the microbiome and the immune response develop concurrently. ${ }^{9-11}$

Antibiotics cause profound changes in the microbiome. ${ }^{12}{ }^{13}$ However, the magnitude of the effect of intrapartum and early-life antibiotics on the breast milk, and the infant oral and intestinal microbiome, and whether effects are only short term or persist long term remain uncertain. Preliminary studies suggest that disruption of intestinal microbiome in the early-life period is associated with the development of a number of immune-mediated and non-immune-mediated diseases, including allergies, ${ }^{14}$ eczema, ${ }^{14}$ asthma, ${ }^{14}$ chronic inflammatory bowel disease ${ }^{15}$ obesity ${ }^{16}$ and diabetes mellitus. ${ }^{17}$ Antibiotic exposure in utero and during infancy has been associated with an increased risk for the same diseases ${ }^{18-20}$ and it is likely that the association between antibiotic exposure and the subsequent development of these diseases is mediated through changes in the infant microbiome. However, the features and composition characteristics of the intestinal microbiome associated with the development of these conditions are unclear.

Importantly, antibiotic exposure can also lead to the development of antibiotic resistance, ${ }^{12}$ resulting in infections that are more difficult and costly to treat, often requiring longer duration of hospital stay, and the use of antibiotics with more adverse effects. Currently, in children, there are sparse data available on the effect of antibiotic exposure on the development and persistence of antibiotic resistance in their intestinal microbiome.

In this prospective cohort study, we will use metagenomic sequencing to determine (1) the effect of intrapartum antibiotics on the composition of the breast milk, and the infant oral and intestinal microbiome (including the development and persistence of antibiotic resistance); (2) the effect of antibiotic exposure in the first year of life on the composition of the infant oral and intestinal microbiome (including the development and persistence of antibiotic resistance); (3) the effect of disruption of the infant oral and intestinal microbiome on health outcomes and (4) determine the compositional overlap between the maternal intestinal microbiome, the breast milk microbiome and the infant oral and intestinal microbiome.

Determining the relationship between antibiotic exposure and changes in the breast milk, and the infant oral and intestinal microbiome, and their potential association with adverse health outcomes will provide stronger evidence for strict antibiotic stewardship. Additionally, it will form the basis for designing studies to investigate interventions to prevent adverse outcomes in situations where antibiotics cannot be avoided, including modifying the intestinal microbiome with directed prebiotics and probiotics or bacteriophages.

\section{OBJECTIVES}

\section{Methods and analysis}

Study design

A prospective single-centre cohort study of 400 motherinfant pairs.

\section{Aims}

Aim 1

To determine the extent to which, and for how long, intrapartum antibiotics affect the composition of the breast milk microbiome and the infant oral and intestinal microbiome, as well as the prevalence of antibioticresistance genes.

\section{Aim 2}

To determine the extent to which, and for how long, antibiotics in the first year of life affect the composition of the oral and intestinal microbiome in infants, as well as the prevalence of antibiotic-resistance genes.

\section{Aim 3}

To determine health outcomes (table 1) in children up to the age of 2 years who have or have not been exposed to intrapartum antibiotics or antibiotics in the first year of life and determine whether there is an association with the composition of the oral and intestinal microbiome.

\section{Aim 4}

To determine the degree to which the maternal intestinal and the breast milk microbiome affect the composition of the infant oral intestinal microbiome and the prevalence of antibiotic resistance genes.

\section{Aim 5}

To determine the degree to which the infant oral microbiome affects the composition of the breast milk microbiome.

\section{Outcomes}

\section{Primary endpoints}

- Composition of the maternal intestinal and breast milk microbiome, and the infant oral and intestinal

Table 1 Clinical outcomes for aim 3

\begin{tabular}{lll}
\hline Outcome & Main measure & Timing \\
\hline Lower respiratory tract illness & No of episodes and hospitalisations ${ }^{2324}$ & First 2 years of life \\
Acute otitis media & No of episodes 2324 years of life \\
Allergic (atopic) sensitisation & Prevalence (positive skin prick test) & First 2 At 2 years of age \\
Eczema & Prevalence (Williams criteria) & At 1 and 2 years of age \\
Weight & Centile (WHO Child Growth Standards) & At 1 and 2 years of age \\
\hline
\end{tabular}


microbiome and the prevalence of antibiotic resistance genes within the infant microbiome at birth and when infants are 7 days, 1, 2, 4, 6, 12 and 24 months of age.

- Number of episodes of lower respiratory tract illnesses (LRTI) and acute otitis media (AOM) in the first 2 years of life.

- Prevalence of allergic sensitisation and eczema at 1 and 2 years of age.

- Weight at 1 and 2 years of age.

\section{Setting and participants}

Sampling frame and study sample

Women who give birth at the Hôpital fribourgeois (HFR) in Fribourg, Switzerland and their infants will be followed over a 2-year period. If recruitment is slow, a second study site will be added.

\section{Recruitment}

Pregnant women attending the antenatal clinic will be given information about the study by a research study nurse or doctor and asked to consider enrolling themselves and their infant in the study. Either both parents or only mothers are present during the antenatal consent interview. We explicitly encourage caregivers to discuss participation with their partners, other family members, doctors and midwives (this is clearly stated in the consent form). We will re-evaluate the willingness to participate when mothers are admitted to the hospital for delivery. Paediatricians will be informed about the children's participation in the study.

\section{Blinding of outcome assessment}

Doctors and study nurses will be blinded to the group of infants (control or antibiotic exposed) when outcomes are measured.

\section{Patient and public involvement}

Patients and public were not involved in the design of this study. The results of this study will be disseminated to parents of the study participants via a participant newsletter distributed by email.

\section{Eligibility criteria}

Inclusion criteria

Healthy infants born at 37 weeks or more gestation who are breastfed.

Mothers will be asked at an antenatal consent interview if they intend to breastfeed. This will be reassessed at delivery. Mothers will only be included if they breastfeed their infants. However, if breast feeding is stopped before the infant reaches 6 months of age, this will not be a reason for exclusion. All the breast milk samples up to that point, as well as stool samples and oral swabs collected afterwards will be analysed.

\section{Exclusion criteria}

Women with the following criteria: HIV, hepatitis B or C infection or unscreened for these infections, antibiotics or probiotics in pregnancy or postpartum period other than during delivery. Infants with the following criteria: low birth weight $(<2500 \mathrm{~g})$ or severe congenital abnormality.

\section{Study outcome measures}

We will use internationally accepted validated measures for clinical outcomes. The study protocol is depicted in table 2 .

\section{Diary}

Parents will be given a structured diary where they can record information about their infant's diet (introduction of formula and new foods), illnesses, medical visits, hospital admissions, use of antibiotics and other medications. This will help them when filling in the questionnaires at the required time points.

\section{Questionnaire}

We will do computer-assisted interviews at birth, and when infants are 7 days, 2, 4, 6, 12 and 24 months of age using best practice international protocols. The following

\begin{tabular}{|c|c|c|c|c|c|c|c|c|}
\hline Time & Birth & 7 days & 1 month & 2 months & 4 months & 6 months & 12 months & 24 months \\
\hline Diary & & $\checkmark$ & \multirow[t]{4}{*}{$\checkmark$} & $\checkmark$ & $\checkmark$ & $\checkmark$ & $\checkmark$ & $\checkmark$ \\
\hline Questionnaire & & $\checkmark$ & & $\checkmark$ & $\checkmark$ & $\checkmark$ & $\checkmark$ & $\checkmark$ \\
\hline Maternal blood sample & 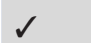 & & & & & & & \\
\hline Maternal stool sample & $\checkmark$ & & & & & & & \\
\hline Breast milk sample & $J^{\text {col }}$ & $\checkmark$ & $\checkmark$ & $\checkmark$ & $\checkmark$ & $\boldsymbol{J}^{*}$ & & \\
\hline Infant oral swab & $\checkmark$ & $\checkmark$ & $\checkmark$ & $\checkmark$ & $\checkmark$ & $\checkmark$ & & \\
\hline Infant stool sample & $\boldsymbol{J}^{\mathrm{mec}}$ & $\checkmark$ & $\checkmark$ & $\checkmark$ & $\checkmark$ & $\checkmark$ & $\checkmark$ & $\checkmark$ \\
\hline Clinical examination & & & & & & & $\checkmark$ & $\checkmark$ \\
\hline Skin prick test (optional) & & & & & & & & $\checkmark$ \\
\hline Blood sampling (optional) & $\boldsymbol{J}^{\mathrm{cb}}$ & & & & & & $\checkmark$ & $\checkmark$ \\
\hline
\end{tabular}

${ }^{*}$ Or before breast feeding is discontinued if earlier than $6 \mathrm{~m}$. cb, cord blood; col, colostrum; mec, meconium. 
data will be recorded: demographic variables including parental ancestry and education, family history of atopy, eczema, asthma and other immune disorders, antenatal variables such as maternal age, weight, smoking habits, underlying diseases, medication and supplementation use (eg, probiotics and vitamins). In addition, we will collect data on delivery history, perinatal course (eg, hospitalisation, infections, antibiotics or oxygen administration), breast feeding (including episodes of mastitis and maternal antibiotic and probiotic use), age of introduction of formula and new foods, administration of probiotics and vitamins, use of antibiotics, antacids and other medications, general practitioner and other medical visits, illnesses including infections and hospital admissions, number of siblings, child care attendance, parental smoking habits, pet ownership, suspected food allergy and eczema (presence, medications). Data will be stored using the Research Electronic Data Capture (Consortium) database. ${ }^{21}$

\section{Clinical examination}

Participants will be reviewed at 12 and 24 months of age in a specially designated clinic at the HFR by a study nurse or doctor using a structured interview and clinical eczema assessment.

\section{Weight}

Weight will be assessed during the clinical examination at 12 and 24 months of age. The WHO Child Growth Standards will be used as a reference for percentiles. ${ }^{22}$

\section{LRTI and AOM}

Symptoms of acute LRTI (such cough and wheeze) and the number of episodes of LRTI and AOM will be recorded by parents, and specific questions will be asked in the questionnaires. We will use the definitions for LRTI developed by Oddy et al and Kusel $e t a l^{2324}$

\section{Eczema}

Prevalence of eczema at 12 and 24 months of life will be assessed by the Williams' UK diagnostic criteria of the International Study of Asthma and Allergy in Childhood. ${ }^{25}$ This will be assessed by using parent-reported eczema from diary information and from the clinical examinations at 12 and 24 months of age. We will assess the severity of eczema using the scoring atopic dermatitis (SCORAD).${ }^{26}$ We will also collect data on age of onset of eczema, distribution of eczema, use of eczema medications and medical consultations and hospital admissions.

\section{Skin prick tests}

Sensitisation to the following panel of allergens will be assessed at 24 months of age in children whose parents consent to this component of the study: cow's milk, egg, peanut, sesame, house dust mite (Dermatophagoides pteronyssinus 1), cat, dog and grass pollen. Skin prick allergy testing will be performed according to standard guidelines. ${ }^{27}$ A positive skin prick test will be defined as an average weal diameter at least $3 \mathrm{~mm}$ greater than that produced by a negative control solution at $15 \mathrm{~min} .{ }^{27}$

\section{Blood sampling}

We will collect maternal blood at time of delivery. We also will obtain cord blood at birth. During the clinical examination at 12 and 24 months of age, blood will be collected by personnel trained in infant venipuncture, whose parents consent to this component of the study. The $5-10 \mathrm{~mL}$ volume required is safe and within limits for weight recommended by the US-based Office of Human Research Protections guidelines for blood collection from healthy infants.

\section{Breast milk samples}

Study nurses will collect one colostrum sample as soon as possible after birth. Mothers will be asked to collect breast milk (with date and time recorded) from their first feed of the day (a minimum of 2 hours required to the previous feed) when infants are 7 days, 1, 2, 4 and 6 months old. If breast feeding is ceased earlier than 6 months, breast milk will be collected before breast feeding is discontinued. Mothers will be instructed to wash their hands and breasts meticulously and to then extract breast milk manually without touching the areola. The first few drops will be discarded. Reminders will be sent by email. Sterile containers will be provided.

\section{Oral swabs}

Oral swabs will be taken from infants as soon as possible after birth by a study nurse. Additionally, parents will then be asked to collect buccal swabs (with date and time recorded) before the first feed of the day when infants are 7 days, 1, 2, 4 and 6 months old. Sterile containers will be provided. Reminders will be sent by email.

\section{Stool samples}

Mothers will be asked to collect stool on or after the day of the delivery (with date and time recorded). A meconium sample will be collected from infants as soon as possible after birth by a study nurse. Parents will then be asked to collect stool samples from their infants when they are 7 days, 1, 2, 4, 6, 12 and 24 months old. Reminders will be sent by email. To minimise variation, parents are asked to collect stool from the first bowel movement of the day (with date and time recorded). Sterile containers will be provided.

\section{Storage of samples}

Parents will be instructed to freeze breast milk, oral swabs and stool samples in sealed bags in their domestic freezer at $-20^{\circ} \mathrm{C}$ until collection by the research team. Samples will be kept frozen during transportation to the laboratory where they will be aliquoted and stored at $-80^{\circ} \mathrm{C}$.

\section{DNA extraction and sequencing}

DNA from breast milk, oral swabs and stool samples (approximately $200 \mathrm{mg}$ ) will be extracted using the FastDNA Spin Kit for soil (MP Biomedicals, Santa Ana, 
California, USA). DNA concentrations will be quantified using a Qubit 4.0 fluorometer (ThermoFisher Scientific, Waltham, Massachusetts, USA) and high sensitivity DNA reagents. Bacterial DNA will be quantified by broad-range bacterial quantitative polymerase chain reaction (qPCR). The library preparation will be done using Nextera DNA Flex Library Preparations Kits. Extracted DNA will be indexed with IDT Illumina Nextera DNA Unique Dual Indexes to allow analysis of pooled samples. $150 \mathrm{bp}$ pair-end sequencing will be done using the Illumina NextSeq. The required sequencing depth to provide adequate coverage of microbial communities for taxonomic profiling will be determined by rarefaction curves. We will aim for a minimum yield of $2-5 \times 10^{6}$ read-pairs per sample. Appropriate negative controls (including controls from sterile containers, extraction kits, etc) and positive controls of mock communities will be included. These controls will be sequenced together with the samples to identify potential environmental and laboratory contaminants.

Researchers carrying out the microbial analyses will be blinded to the group identity of infants (control or antibiotic exposed group).

\section{Storage of blood samples}

Peripheral blood mononuclear cells will be separated from whole blood and stored in liquid nitrogen. Plasma will be stored frozen at $-80^{\circ} \mathrm{C}$. These will be retained for future analysis in projects to evaluate the effect of microbiome on the immune system, including immunophenotyping and analysis of markers of immune function.

\section{Statistical power calculation}

The analysis for the a priori computation of the required sample size was conducted using the G*Power V.3.1 software. For the power analysis, the repeated measurements multivariate analysis of variances (ANOVAs) were considered. The calculation was based on F-tests for the interaction effect of the between-subject factor antibiotics vs no antibiotics and the within-subject factor time (eight time points). We chose a small to medium effect size of 0.22 for the calculation. With a significance level of $5 \%$ per test and a power of $80 \%$ the power analysis yields a sample size of 304 . While we will attempt to retain and follow-up all participants, we are powering our study on 'a worst-case scenario' assumption that complete 24-month data will be available for $76 \%$ of participants (this corresponds to a drop-out rate of $12 \%$ per year). We will, therefore, recruit approximately 400 infants in total (plus their mothers, therefore 800 participants). Approximately $40 \%-50 \%$ of infants are exposed to prophylactic intrapartum antibiotics at our institution for colonisation with Group B streptococcus (GBS) or premature rupture of membranes. Additional 50\%-60\% of children are expected to be exposed to antibiotics during the first 2 years of life.

\section{Bioinformatics}

Sequences will be trimmed according to quality scores and sequencing adaptors will be removed using Trimmomatic. ${ }^{28}$ Host sequences will be removed by mapping against the Human genome with Bowtie2. ${ }^{29}$ High-quality sequences will be used to create taxonomic and functional profiles using MetaphlAn2 ${ }^{30}$ and HUMAnN2, ${ }^{31}$ respectively. Antibiotic resistance genes will be identified using ResFinder. ${ }^{32}$ We will share our metagenomic data through the European Nucleotide Archive.

\section{Statistical analysis \\ Considered metrics \\ Alpha-diversity}

Alpha-diversity indexes are descriptive of the intra-sample richness (number of taxonomic functional features), evenness (features distribution) or diversity (richness weighted by evenness). ${ }^{33}$ We will use Chao richness (number of different taxa) and Simpson diversity (distribution of taxa) to summarise the alpha-diversity for each sample.

\section{Beta-diversity}

For beta-diversity, samples are compared for their composition in features of interest (taxonomic or functional units). ${ }^{34}$ All-versus-all distances between samples are computed in pairwise comparisons and summarised in distance matrices. We will use indices, with distance equal to 1-index value, to capture different dimensions of microbial structures, ${ }^{34}$ restricting ourselves to non-Euclidean indices and excluding the 'joint absences' as sharing of unseen features would have doubtful significance in this context. Feature presence/absence will be described by Jaccard indices and quantitative overlap by Bray-Curtis indices. In taxonomic comparisons, Unifrac (presence/ absence) and weighted Unifrac (quantitative) will be used to account for features phylogenetic distances. ${ }^{35}$ Statistics on distances matrices require adapted methods to assess for significant differences in average location (centroid) of the samples of groups of interest. For this, we will use the permutational multivariate analysis of variance (PERMANOVA) approach implemented in the Adonis2 function of the vegan $R$ package. PERMANOVA can be sensitive to variance heterogeneity in unbalances groups. Thus, variance homogeneity will be tested by ANOVA to centroids.

\section{Differential abundance testing}

Metagenomics data are compositional due to technical limitations. ${ }^{36}{ }^{37}$ Relative abundances of bacterial and non-bacterial (archaeal, eukaryotic and viral) taxa and antibiotic resistance genes, will be directly integrated for analyses. For bacteria, we will transform observed proportions into absolute quantities by multiplying proportions (\%) by measured microbial loads, quantified by broadrange qPCR. Thus, we will explore correlation abundance testing of transformed counts for taxa and bacterial resistance genes. Statistical challenges of metagenomics data 
are the high number of features (and related multipletesting false discovery) and features sparsity (a given features-species or genes-will be observed only in a few samples, leading to a high proportion of zeros in count tables). These limitations in metagenomics statistics were only recently recognised and the developments of methods accounting for these is a field of active research and publication. Thus, we will follow on future developments, recommendation and consensuses in the field regarding these challenges. Currently, identified solutions accounting for these limitations are MetagenomSeq, Aldex2 and Maaslin2, which all integrate normalisation and correlation testing to account for the prementioned limitations while trying to identify differentially abundant features (genes or species) between tested groups.

\section{Statistical plan for aim 1 and 2}

Antibiotics exposed samples will be compared with non-exposed samples (grouped per received drug) for differences in alpha-diversity metrics and abundances of bacterial and non-bacterial taxa and antibiotic resistance genes with significant changes defined as a $>0.5 \%$ change in abundance between groups. Alpha-diversity metrics are continuous numeric values. Normality of the data will be checked by the Kolmogorov-Smirnov test and QQ-plots. For normal variables, a one-way ANOVA will be used. If normality cannot be reached by transformation, differences between groups will be analysed using a non-parametric Kruskal-Wallis test, or in case of pairwise comparisons, a Mann-Whitney U test. For comparison of abundance Pearson $x^{2}$ tests will be used. Infant age, demographics, delivery mode, feeding method and antibiotic type and dose will be modelised in (PERMANOVA, adonis2 in VEGAN package, R Foundation) using betadiversity distance matrices to identify significant contributors to the microbiome composition.

\section{Statistical plan for aim 3}

For integrated analysis of the microbiome data and clinical outcomes, allergic sensitisation, eczema and overweight cases will be defined using the prospectively collected outcome measures. The relationship between alpha-diversity and clinical outcomes will be investigated using logistic regression. The relationship between taxa and these clinical outcomes will be determined by hierarchical clustering of communities using heatmaps and principal component analysis. Significance of grouping of clinical categories using PERMANOVA. Microbes that have significantly different abundance between the clinical outcome groups will be identified using Aldex $2 .{ }^{38}$ The potential influence of antenatal and postnatal factors on the microbiome or clinical outcomes will be accounted for in all analyses by PERMANOVA and unsupervised hierarchical clustering.

\section{Statistical plan for aim 4}

To compare paired maternal stool, breast milk and infant stool samples, we will do clustering analysis and perform a Wilcoxon rank sum test on beta-diversity distances between true mother-infant pairs and randomly paired mothers and infants matched by infant age. These different analyses should allow to describe the relationships between samples of different origins and identify determinants of microbiome composition.

To further characterise the mother-to-infant microbiome transmission, the StrainPhlAn software will be used. $^{39}$ This software enables same-species sequence comparisons at the single nucleotide polymorphism level to define sample-specific strains. Combined results for shared species allow precise description of the phylogenetic distance between samples. Added to the betadiversity analyses, this will allow to better disentangle the hypothesised seeding from maternal stool and breast milk to the infant oral and stool microbiome.

\section{Statistical plan for aim 5}

The same as for aim 4 will be done for comparison of the breast milk and the infant oral microbiome.

\section{Missing data}

If the fraction of missing data is less than $5 \%$, the primary analysis will be a complete case analysis. If not, the rate and patterns of missing data will be examined and, if appropriate, multiple imputation models will be applied for the outcome variables.

\section{ETHICS AND DISSEMINATION \\ Ethics approval}

The ABERRANT study has been approved by the commission cantonale d'éthique de la recherche sur l'être humain (CER-VD) du Canton de Vaud (\#2019-01567), Switzerland.

\section{Recruitment and consent}

Written informed consent will be obtained from all participants included in the trial. Participants will be informed that they are not obliged to take part in the study and are free to withdraw at any time without any impact on their future care.

\section{Data collection and storage}

Data will be sourced from medical maternal and infant records, as well as by questionnaire from parents. Data will be deidentified and entered in to a secure, web-based electronic database.

\section{Safety}

No serious adverse reactions are anticipated but these will be checked for by the data safety and monitoring committee.

\section{Dissemination of results}

Outcomes will be disseminated through publication according to the Standard Protocol Items: Recommendations for Interventional Trials statement and will be presented at scientific conferences. 


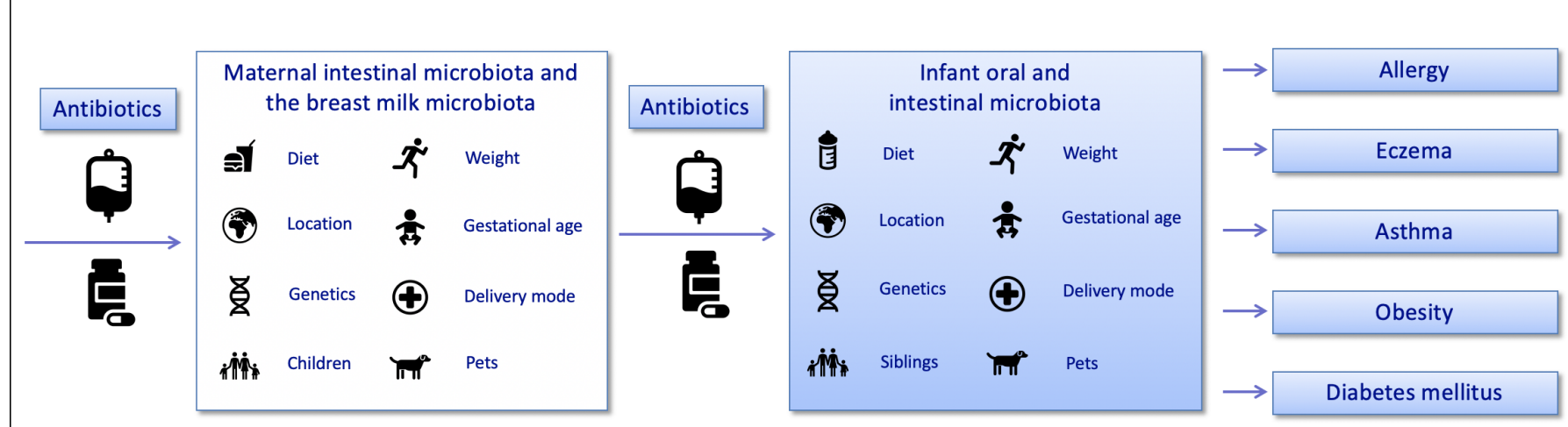

Figure 1 Summary of factors that might influence the composition of the maternal intestinal and breast milk microbiome, and the infant oral and intestinal microbiome together with possible associated adverse health outcomes.

\section{Study duration}

We aim to recruit participants over a 2-year period.

\section{DISCUSSION}

The intestinal microbiome is crucial in the development of the immune system and regulation of immune responses, especially during infancy, when the intestinal microbiome and the immune response develop concurrently. ${ }^{40}$ The development of intestinal microbiome is easily disrupted by external factors and perturbation during this vulnerable period may have a large influence on immune development. A number of factors influence the development of the infant intestinal microbiome, including gestational age, delivery mode, feeding, and maternal and infant antibiotic exposure (figure 1). ${ }^{41}$ While the effect of delivery mode and feeding methods on the establishment of microbial communities has been well studied, much less is known about the effects of intrapartum and early-life antibiotic exposure on the establishment of microbial communities in the intestinal microbiome. $^{13}$

A number of commonly used antibiotics have profound effects on specific bacteria within the intestinal microbiome, as detailed in a recent systematic review. ${ }^{12}$ This 'collateral damage' includes changes in diversity and abundance of microbial taxa, a decrease in 'colonisation resistance' (protection against colonisation with potentially pathogenic organisms) and the development of antibiotic resistance. To date, most studies on the effect of antibiotic exposure on the intestinal microbiome have been done in adults. ${ }^{12}$ The main findings of these studies are that antibiotics often lead to a decreased bacterial diversity, a decrease in the abundance of anaerobic bacteria, an increase in abundance of Enterobacteriaceae other than Escherichia coli and an increase in the abundance of yeast. ${ }^{12}$ These studies show that changes in the intestinal microbiome after just one course of antibiotics can persist up to 4 years. ${ }^{12}$ However, the clinical consequences of changes in the composition of the intestinal microbiome with antibiotic treatment are unknown. An increase in Enterobacteriaceae, which are often resistant to beta-lactam and other antibiotics, might render the host more susceptible to infections with antibioticresistant bacteria. This phenomenon has been observed in infants in neonatal intensive care units, who become more often colonised with Klebsiella spp., Enterobacter spp. and Citrobacter spp., when treated with antibiotics. ${ }^{42}$

Intrapartum antibiotic prophylaxis (IAP) has become common practice in obstetric medicine and is used in up to $40 \%$ of deliveries, which makes it the most common source of antibiotic exposure in neonates. ${ }^{1}$ IAP is routinely used in both elective and emergency caesarean section. It is also routinely used in women who are colonised with GBS. Despite the benefits, the risks associated with exposing a large number of infants to antibiotics, especially the long-term effects on health through changes in the microbiome, remain unclear. Infants who were exposed to IAP have been reported to have a lower alphadiversity, a lower relative abundance of actinobacteria, especially Bifidobacteriaceae, and a larger relative abundance of proteobacteria in their intestinal microbiome compared with non-exposed infants. ${ }^{13}$ Furthermore, they have been reported to have a higher number of betalactamase encoding genes. ${ }^{43}$

Breast feeding has been shown to be beneficial in preventing many communicable and non-communicable diseases. ${ }^{44}$ Despite intensive research into the positive health effects of breast feeding, the underlying mechanisms are still not understood. However, a large part of the beneficial effects of breast milk is likely mediated through the microbiome and its associated immunomodulatory, anti-inflammatory and antimicrobial components. The breast milk microbiome also likely plays a part in shaping the infant's intestinal microbiome, ${ }^{5}$ to which extent we will be investigated in this study.

There is relatively little data about the effects of IAP on the composition of the breast milk microbiome. ${ }^{45-47}$ Mothers who receive IAP have been reported to have a lower abundance or even an absence of the beneficial bacteria Bifidobacterium spp. in their breast milk. ${ }^{45}$ Furthermore, 
in a small study IAP has been shown to increase antibiotic resistance genes in the breast milk microbiome. ${ }^{48}$ There is also some evidence suggesting that mothers who receive IAP have a higher bacterial richness and diversity in their breast milk microbiome compared with mothers who do not receive antibiotics. ${ }^{45}$ However, these findings have to be interpreted with caution: it could be that antibiotics lead to lower bacterial numbers and therefore signals from contamination, for example, bacteria found in DNA extraction or sequencing kits might be amplified more leading to a the detection of a higher diversity. Use of broad-range qPCR to quantity bacterial load in milk samples will allow to assess this potential bias.

Interestingly, recent preliminary studies have also shown that delivery mode affects the composition of the breast milk microbiome. ${ }^{45} 474950$ However, during suckling, a high degree of retrograde flow of milk into the mammary ducts can occur, ${ }^{6}$ transferring bacteria from the infant to the mother, as postulated for GBS. ${ }^{51}$ It is therefore possible that the differences in the breast milk microbiome observed with different delivery modes are mediated through differences in the oral microbiome of infants.

This study will determine the effect of intrapartum and early-life antibiotics on the composition of the breast milk, and the infant oral and intestinal microbiome and antibiotic resistance. The knowledge gained by this study will make an important contribution to the growing field of research investigating the importance of the immunological role of the breast milk microbiome and the infant intestinal microbiome on infant health. It will form the basis for investigating the interplay between the microbiome and the regulation of the human immune system and possible adverse health outcomes, such as the development of immune-mediated diseases and non-immunemediated diseases, including allergic diseases.

The results of this study will also build a stronger evidence base for strict antibiotic stewardship and form the basis for development of evidence-based interventions to prevent adverse outcomes in situations where antibiotics cannot be avoided, including modifying the intestinal microbiome with directed prebiotics and probiotics or bacteriophages.

\section{Author affiliations}

${ }^{1}$ Faculty of Science and Medicine, University of Fribourg, Fribourg, Switzerland ${ }^{2}$ Department of Paediatrics, Fribourg Hospital HFR, Fribourg, Switzerland

${ }^{3}$ Institute of Microbiology, Lausanne University Hospital, Lausanne, Switzerland

${ }^{4}$ Facultiy of Medicine, University of Lausanne, Lausanne, Switzerland

${ }^{5}$ Microbiology Laboratory, Fribourg Hospital HFR, Fribourg, Switzerland

${ }^{6}$ Department of Biology, University of Fribourg, Fribourg, Switzerland

${ }^{7}$ Swiss Institute of Bioinformatics, Lausanne, Switzerland

${ }^{8}$ Department of Paediatrics, The University of Melbourne, Parkville, Victoria, Australia

${ }^{9}$ Infectious Diseases Research Group, Murdoch Children's Research Institute,

Parkville, Victoria, Australia

${ }^{10}$ Infectious Diseases Unit, The Royal Children's Hospital Melbourne, Parkville, Victoria, Australia
Contributors $\mathrm{PZ}$ is the lead investigator. VS, LF, NC and PZ were responsible for study conception and design. JW, AL-B and PZ were responsible for funding acquisition and implementation. MV drafted the manuscript and coordinated the manuscript preparation and revision. PZ and VS have developed the statistical analysis plan. MV and PZ have developed the online questionnaires and database setup in REDCap. WJ will be responsible for sample analysis. MV, VS, WJ, DB, VD-M, AL-B, JW, LF and NC provided critical evaluation and revision of manuscript and have given final approval of the manuscript accepting responsibility for all aspects.

Funding This trial is funded by the University of Fribourg and the Fribourg Hospital HFR, Switzerland. VS is supported by a SNSF grant (no 10531 C-170280 - LF, G. Greub and F. Taroni).

\section{Competing interests None declared.}

Patient and public involvement Patients and/or the public were not involved in the design, or conduct, or reporting, or dissemination plans of this research.

Patient consent for publication Not required.

Provenance and peer review Not commissioned; externally peer reviewed.

Open access This is an open access article distributed in accordance with the Creative Commons Attribution Non Commercial (CC BY-NC 4.0) license, which permits others to distribute, remix, adapt, build upon this work non-commercially, and license their derivative works on different terms, provided the original work is properly cited, appropriate credit is given, any changes made indicated, and the use is non-commercial. See: http://creativecommons.org/licenses/by-nc/4.0/.

ORCID iD

Petra Zimmermann http://orcid.org/0000-0002-2388-4318

\section{REFERENCES}

1 Stokholm J, Schjørring S, Pedersen L, et al. Prevalence and predictors of antibiotic administration during pregnancy and birth. PLoS One 2013;8:e82932.

2 Zimmermann P, Curtis N. Prophylactic antibiotics after operative vaginal delivery. Lancet 2020;395:189.

3 Hellman J, Grape M, Ternhag A. Antibiotic consumption among a Swedish cohort of children born in 2006. Acta Paediatr 2015;104:1035-8.

4 Anderson $\mathrm{H}$, Vuillermin $\mathrm{P}$, Jachno K, et al. Prevalence and determinants of antibiotic exposure in infants: a population-derived Australian birth cohort study. J Paediatr Child Health 2017;53:942-9.

5 Pannaraj PS, Li F, Cerini C, et al. Association between breast milk bacterial communities and establishment and development of the infant gut microbiome. JAMA Pediatr 2017;171:647-54.

6 Ramsay DT, Kent JC, Owens RA, et al. Ultrasound imaging of milk ejection in the breast of lactating women. Pediatrics 2004;113:361-7.

7 Perez PF, Doré J, Leclerc M, et al. Bacterial imprinting of the neonatal immune system: lessons from maternal cells? Pediatrics 2007;119:e724-32

8 Jost T, Lacroix C, Braegger CP, et al. Vertical mother-neonate transfer of maternal gut bacteria via breastfeeding. Environ Microbiol 2014;16:2891-904

9 Fujimura KE, Lynch SV. Microbiota in allergy and asthma and the emerging relationship with the gut microbiome. Cell Host Microbe 2015;17:592-602.

10 Panzer AR, Lynch SV. Influence and effect of the human microbiome in allergy and asthma. Curr Opin Rheumatol 2015;27:373-80.

11 Coit P, Sawalha AH. The human microbiome in rheumatic autoimmune diseases: a comprehensive review. Clin Immunol 2016;170:70-9.

12 Zimmermann P, Curtis N. The effect of antibiotics on the composition of the intestinal microbiota - a systematic review. $J$ Infect 2019;79:471-89.

13 Zimmermann P, Curtis N. Effect of intrapartum antibiotics on the intestinal microbiota of infants: a systematic review. Arch Dis Child Fetal Neonatal Ed 2020;105:201-8.

14 Zimmermann P, Messina N, Mohn WW, et al. Association between the intestinal microbiota and allergic sensitization, eczema, and asthma: A systematic review. J Allergy Clin Immunol 2019;143:467-85.

15 Manichanh C, Rigottier-Gois L, Bonnaud E, et al. Reduced diversity of faecal microbiota in Crohn's disease revealed by a metagenomic approach. Gut 2006;55:205-11.

16 Scott FI, Horton DB, Mamtani R, et al. Administration of antibiotics to children before age 2 years increases risk for childhood obesity. Gastroenterology 2016;151:120-9. 
17 Knip M, Siljander $\mathrm{H}$. The role of the intestinal microbiota in type 1 diabetes mellitus. Nat Rev Endocrinol 2016;12:154-67.

18 Mueller NT, Whyatt R, Hoepner L, et al. Prenatal exposure to antibiotics, cesarean section and risk of childhood obesity. Int $\mathrm{J}$ Obes 2015;39:665-70. (2005).

19 Ong MS, Umetsu DT, Mandl KD. Consequences of antibiotics and infections in infancy: bugs, drugs, and wheezing. Annals of allergy, asthma \& immunology : official publication of the American College of Allergy, Asthma, \&amp. Immunology 2014;112:441-5.

20 Ungaro R, Bernstein CN, Gearry R, et al. Antibiotics associated with increased risk of new-onset Crohn's disease but not ulcerative colitis: a meta-analysis. Am J Gastroenterol 2014;109:1728-38.

21 Harris PA, Taylor R, Thielke R, et al. Research electronic data capture (REDCap)--a metadata-driven methodology and workflow process for providing translational research informatics support. $J$ Biomed Inform 2009;42:377-81.

22 Organization WH. Who child growth standards, 2006. Available: https://www.who.int/childgrowth/standards/en/

23 Oddy WH, de Klerk NH, Sly PD, et al. The effects of respiratory infections, atopy, and breastfeeding on childhood asthma. Eur Respir J 2002;19:899-905.

24 Kusel MMH, de Klerk NH, Holt PG, et al. Role of respiratory viruses in acute upper and lower respiratory tract illness in the first year of life: a birth cohort study. Pediatr Infect Dis J 2006;25:680-6.

25 Williams HC, Burney PG, Pembroke AC, et al. The U.K. Working Party's diagnostic criteria for atopic dermatitis. Br J Dermatol 1994;131:406-16.

26 Pucci N, Novembre E, Cammarata MG, et al. Scoring atopic dermatitis in infants and young children: distinctive features of the SCORAD index. Allergy 2005;60:113-6.

27 Bernstein IL, Storms WW. Practice parameters for allergy diagnostic testing. joint Task force on practice parameters for the diagnosis and treatment of asthma. the American Academy of allergy, asthma and immunology and the American College of allergy, asthma and immunology. Ann Allergy Asthma Immunol 1995;75:543-625.

28 Andrews S. FastQC: a quality control tool for high throughput sequence data, 2010. Available: http:// wwwbioinformaticsbabrahamacuk/projects/fastqc

29 Langmead B, Salzberg SL. Fast gapped-read alignment with Bowtie 2. Nat Methods 2012;9:357-9.

30 Segata N, Waldron L, Ballarini A, et al. Metagenomic microbial community profiling using unique clade-specific marker genes. Nat Methods 2012;9:811-4.

31 Abubucker S, Segata N, Goll J, et al. Metabolic reconstruction for metagenomic data and its application to the human microbiome. PLoS Comput Biol 2012;8:e1002358.

32 Zankari $\mathrm{E}$, Hasman $\mathrm{H}$, Cosentino S, et al. Identification of acquired antimicrobial resistance genes. J Antimicrob Chemother 2012:67:2640-4.

33 Kim B-R, Shin J, Guevarra R, et al. Deciphering diversity indices for a better understanding of microbial communities. J Microbiol Biotechnol 2017;27:2089-93.
34 Anderson MJ, Crist TO, Chase JM, et al. Navigating the multiple meanings of $\beta$ diversity: a roadmap for the practicing ecologist. Ecol Lett 2011;14:19-28.

35 Lozupone CA, Hamady M, Kelley ST, et al. Quantitative and qualitative beta diversity measures lead to different insights into factors that structure microbial communities. Appl Environ Microbiol 2007;73:1576-85.

36 Gloor GB, Macklaim JM, Pawlowsky-Glahn V, et al. Microbiome datasets are compositional: and this is not optional. Front Microbiol 2017;8:2224.

37 Quinn TP, Erb I, Gloor G, et al. A field guide for the compositional analysis of any-omics data. Gigascience 2019;8:giz107.

38 McMurdie PJ, Holmes S. phyloseq: an R package for reproducible interactive analysis and graphics of microbiome census data. PLOS One 2013;8:e61217.

39 Truong DT, Tett A, Pasolli E, et al. Microbial strain-level population structure and genetic diversity from metagenomes. Genome Res 2017;27:626-38.

40 Zeevi D, Korem T, Segal E. Talking about cross-talk: the immune system and the microbiome. Genome Biol 2016;17:50.

41 Zimmermann P, Curtis N. Factors influencing the intestinal microbiome during the first year of life. Pediatr Infect Dis $J$ 2018;37:e315-35.

42 Goldmann DA, Leclair J, Macone A. Bacterial colonization of neonates admitted to an intensive care environment. $J$ Pediatr 1978:93:288-93.

43 Nogacka A, Salazar N, Suárez M, et al. Impact of intrapartum antimicrobial prophylaxis upon the intestinal microbiota and the prevalence of antibiotic resistance genes in vaginally delivered fullterm neonates. Microbiome 2017;5:93.

44 Kelishadi R, Farajian S. The protective effects of breastfeeding on chronic non-communicable diseases in adulthood: a review of evidence. Adv Biomed Res 2014;3:3.

45 Hermansson $\mathrm{H}$, Kumar $\mathrm{H}$, Collado $\mathrm{MC}$, et al. Breast milk microbiota is shaped by mode of delivery and intrapartum antibiotic exposure. Front Nutr 2019;6:4.

46 Soto A, Martín V, Jiménez E, et al. Lactobacilli and bifidobacteria in human breast milk: influence of antibiotherapy and other host and clinical factors. J Pediatr Gastroenterol Nutr 2014;59:78-88.

47 Zimmermann P, Curtis N. Breast milk microbiota: a review of the factors that influence composition. J Infect 2020;S01634453:30063-3

48 Pärnänen K, Karkman A, Hultman J, et al. Maternal gut and breast milk microbiota affect infant gut antibiotic resistome and mobile genetic elements. Nat Commun 2018;9:3891.

49 Khodayar-Pardo P, Mira-Pascual L, Collado MC, et al. Impact of lactation stage, gestational age and mode of delivery on breast milk microbiota. J Perinatol 2014;34:599-605.

50 Toscano M, De Grandi R, Peroni DG, et al. Impact of delivery mode on the colostrum microbiota composition. BMC Microbiol 2017; $17: 205$.

51 Zimmermann P, Gwee A, Curtis N. The controversial role of breast milk in GBS late-onset disease. J Infect 2017;74 Suppl 1:S34-40. 